\title{
Single NGS for Extremely Large Telescope MCAO
}

\author{
Esposito, S. ${ }^{1, a}$, Gori, P-M. ${ }^{1}$, and Brusa, G. ${ }^{1}$ \\ Osservatorio Astrofisico di Arcetri, Lgo E. Fermi n.5 - 50125 Firenze, Italy
}

\begin{abstract}
Most of the MCAO systems planned for astronomical telescopes use laser beacons to create at will a star constellation having the geometry required for a good tomographic reconstruction. The problem of undetermined LGS tip tilt prevents one from measuring the tilt of the reference sources. As a consequence the global tilt of the NGSs over the FoV and the altitude and amplitudes of the low order modes are not correctly estimated. In general to solve these problems several NGSs are used. However the use of a few NGSs can reduce the sky coverage of the LGS MCAO system by a significant factor. In this paper we present a technique to estimate the low order modes including overall Tilt in the tomographic distribution directly from the LGSs and a single NGS. In this way the sky coverage of the MCAO system is improved. This paper reports the performance of this technique for the case of a $42 \mathrm{~m}$ diameter telescope calculated using numerical and analytical simulations.
\end{abstract}

\section{Introduction}

Nowadays, Multi Conjugate Adaptive Optic (MCAO) [2] for the Extremely Large Telescope (ELT) considers using Sodium Laser Guide Star (LGS) as reference star for determining and correcting the atmospheric aberration in the Field of View (FoV) of interest [5], [6].

A typical problem when using LGS is to measure the artificial star Tilt signal.

Briefly, the upward propagation from the ground to the Sodium Layer is passing through the atmosphere. The arrival point of the beam at the Sodium Layer depends on the wavefront perturbations encountered. The principal result of this, is an unknown position of the LGS spot in the Sodium Layer.[11].

Therefore the LGS is forced to be centered on the WFS (WaveFront Sensor) optical axis. In such a way the TT information of each LGS is nulled.

As a consequence, this leads to error of MCAO reconstruction especially for Low Order (LO) modes. Thus LGS's are not sufficient to reconstruct all the Zernike [9] modes of the tomographic volume. That's why, in most of the papers and reports about MCAO there is a need for 3 Natural Guide Stars (NGS) present in the patrol field [12]. Finding 3 NGS's is a strong constraint in terms of Sky Coverage [1], [4]. For instance, the sky coverage with a 2 arcmin FoV, and a limit magnitude of 17 at the North Galactic Pole is about $31 \%$, with one required NGS, and drops to $2.9 \%$ with 3 required NGS's. Actually another requirement is the NGS's distribution in the sky. In effect, having 3 NGS's far one to one another is essential.

In this paper we propose a technique to reduce the number of NGS's needed to only one. We first explain the problem and detail the principle of our technique. Then we present some numerical simulation first results done for the ELT case and we conclude by summarizing the problems encountered, and opening the discussion for the future of this method.

\section{Technique concept}

As described above, the TT of the LGS is unknown [11]. This introduces error in the reconstructed Low Order (LO) modes and at a lower level in High Order (HO) modes too. This is shown quantitatively in Fig.1. We simulated MCAO reconstruction in the following configuration : 4 LGS's, a FoV of 2 arcmin, a telescope diameter of $42 \mathrm{~m}, 2$ layers $(1 \mathrm{~km} \& 8 \mathrm{~km}), 2$ DM's. The DM's are supposed to be

\footnotetext{
a e-mail: esposito@arcetri.astro.it
} the original work is properly cited. 
exactly conjugated to the atmospheric layer. This configuration will be used for all the results presented in this paper. We specify here that in this simulations we used geometrical conical propagation for the LGS and cilindrical propagation for the NGS.

We report in Fig. 1 the residual error variance for the MCAO reconstruction when the LGS Tip-Tilt is set to zero before matrix multiplication. We notice that MCAO correction is not achieved, neither for LO modes and at a lower level nor for HO modes.

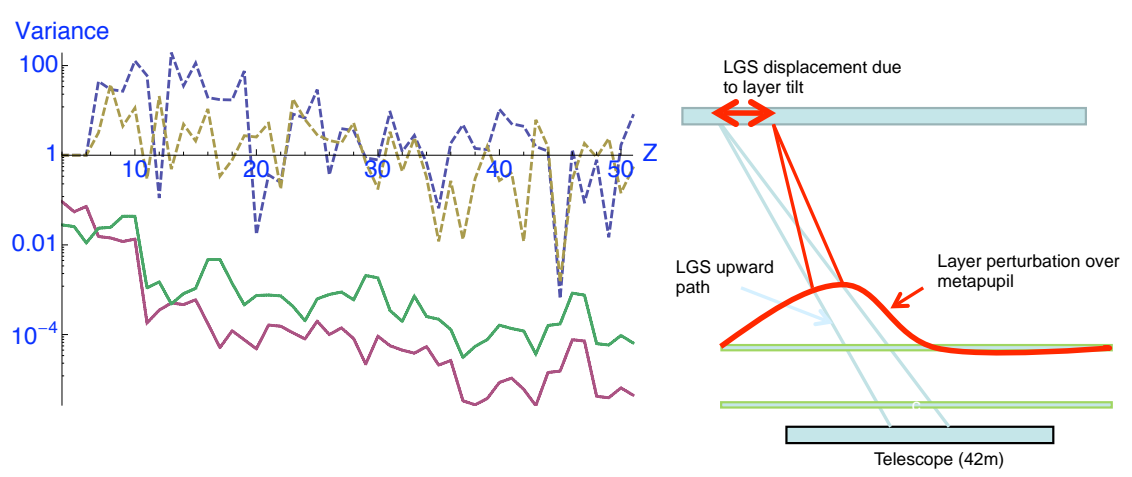

Fig. 1. Residual Error due to LGS jitter. On the right is drawn the component of the upward TT of the LGS. The upward beam does not hit the nominal position on the Sodium layer because of TT encountered during the upward propagation. On the left the plot shows residual error variance after reconstruction when TT is not measured on the WFS. Dashed lines are the Zernike coefficients for both atmosphere layer. Solid lines are the residual variances for both DM's. The vertical axis represents variance in $\mathrm{rad}^{2}$ at the Sodium Laser wavelength; the horizontal axis is the Zernike mode number. One can notice that removing TT affects reconstruction of higher modes too.

In the following section, we propose a new method to solve this problem considering the TT information of LGS's.

The technique concept consists of launching the LGS in such a way that the upward beam is propagating through the tomographic volume. In this way, we can take into account the upward propagation for tomographic reconstruction. Briefly this is done by computing the LGS spot displacement on the sodium layer due to each atmospheric layer perturbation (see fig.1 right). The TT part of the upward wavefront turbulence is needed: in fact only the spatial position of the backscattered beam on the sodium layer affects the LGS WFS's. Thus we will only consider the TT component of the LGS beam during the upward propagation.

As a consequence of this method, the TT of the LGS's is now measured on the WFS. This helps to reconstruct low order modes from LGS's.

Still the Isoplanatic TT of the atmosphere remains undetermined from LGS Tilt measurement, one NGS is needed [3].

\section{The new IM}

In this work the WFS's measurements are simulated by directly decomposing the wavefront received by the WFS in Zernike modes.So,

The Interaction Matrix (IM) is built projecting the Zernike modes applied in each metapupil onto measured modes. A block of the MCAO IM (1 star; $1 \mathrm{DM})$ is shown on Fig.2 (A-left). The $\left(C_{i, j}\right)_{k}$ coefficient of the downward matrix is the amount of Zernike $j$ measured by the WFS when a unitary $Z_{i}$ is applied in the DM $k$. The $\left(C_{2, j}\right)_{k}$ or $\left(C_{3, j}\right)_{k}$ coefficient of the upward matrix is the amount of Tip or Tilt measured by the WFS when a unitary $Z_{i}$ is applied in the DM $k$.

In our specific case, we add to the downward reconstructor an upward TT reconstructor on the first 2 lines of the IM (first line for tip and second for tilt as seen on Fig.2 (A-central)). The new IM is 
(A)

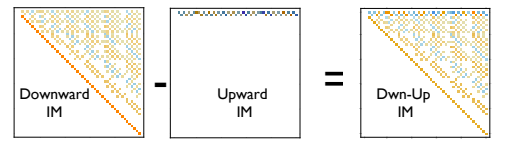
... 4 LGS's, 2 DM's =>

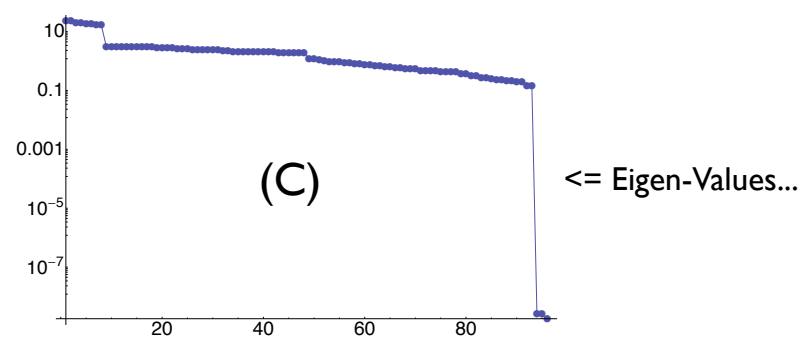

(B)

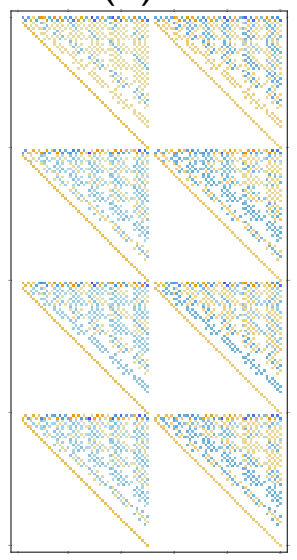

Fig. 2. New Interaction Matrix. (A) The new IM is composed of an upward (A - center) and a downward propagation matrix. (A - left). The up matrix is made up of 2 only lines because we take into account only TT. The big Matrix (B) represents the extension of the new IM for 2 DM ( 2 block columns) and 4 LGS's ( 4 block lines). The plot (C) shows the eigenvalues of (B). The vertical axis is the eigenvalue, in logarithmic scale, and the horizontal axis is a metapupil mode number. We can see that there are 3 null eigenvalues.

obtained in the case of launching LGS's from inside the pupil, taking into account footprint dimensions at each reconstructed layer.

Then the IM, shown in Fig.2 (A-right), is extended to 2 layers, 4 LGS's as seen on Fig.2 (B). This IM becomes inverted to form the Reconstruction Matrix (RM). On Fig.2 (C) is plotted the eigenvalues of the RM. We notice that 3 of them are null. This corresponds to 3 unseen modes involving: $Z_{4}, Z_{5}$ and $Z_{6}$. We show in the next section the main effect of this 3 null eigenvalues.

\section{Results for Radial order higher than 2}

We remind that numerical simulations has been done with the configuration detailed in Sec. 2. In this simulation the wavefront analysis allows to reconstruct the wavefront starting from $Z_{7}$ without errors on other modes. As reported in Fig.3, the residual error variance for both layers and all modes except

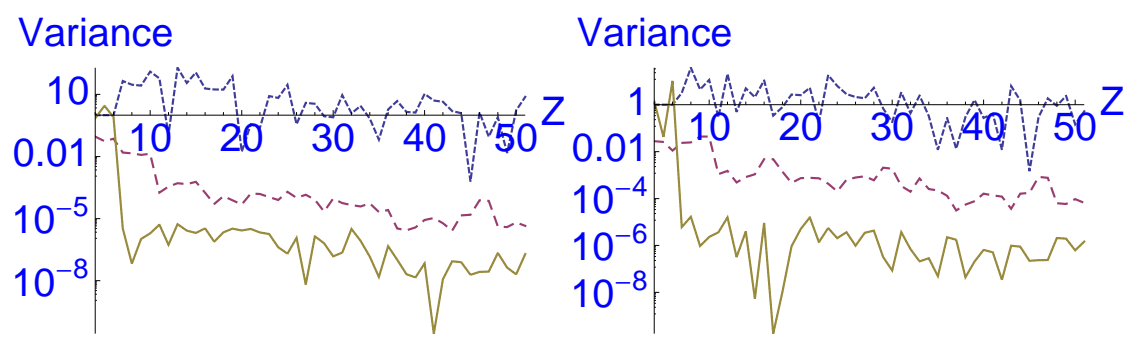

Fig. 3. Residual error considering upward TT. Both plots show the residual error variance after correction in $\mathrm{rad}^{2}$ on a logarithmic scale vs Zernike mode number. The left and right plot are for the DM's conjugated to the atmosphere layer respectively at $1 \mathrm{~km}$ and $8 \mathrm{~km}$. The top dashed lines represent Zernike coefficients of the atmospheric turbulence. Solid lines the residual error variance after correction. The large dashed line is the residual shown on Fig.1 (when TT is not measured).

for $Z_{4}$ to $Z_{6}$ is very small. We show in Sec 5 how we correct these 3 modes. In particular we note that 
the error when upward Tilt is considered is about 2 order of magnitude smaller than the case of Fig.1 when LGS TT is neglected.

\section{Radial order 2}

The NGS WFS data that measures from $Z_{2}$ to $Z_{6}$, are used to determine tomographic Zernike modes $Z_{4}, Z_{5}$ and $Z_{6}$ (radial order $n=2$ ) plus the isoplanatic TT. We note that NGS footprints doesn't have the same dimensions as the LGS on a given layer. This condition allow us to write 2 independent equations for each one of $Z_{4}, Z_{5}$ and $Z_{6}$. These equations (Eq.1 and Eq.2) relates the total amplitude of a given Zernike mode seen by the NGS and the LGS respectively.

$$
\begin{aligned}
& \left(Z_{J}\right)_{N G S}-\tilde{C}_{C i l}\left(Z_{J}\right)=\alpha_{N G S} Z_{J_{1}}+\beta_{N G S} Z_{J_{2}} \\
& \left(Z_{J}\right)_{L G S}-\tilde{C}_{C o n}\left(Z_{J}\right)=\alpha_{L G S} Z_{J_{1}}+\beta_{L G S} Z_{J_{2}}
\end{aligned}
$$

Because each of these modes does not give any projection on the other two, the equations are decoupled. For instance for $J=4, \alpha$ and $\beta$ are coefficient projecting metatpupil focus on focus over LGS and NGS footprint. $\tilde{C}_{C i l}\left(Z_{4}\right), \tilde{C}_{C o n}\left(Z_{4}\right)$ are the contributions to measured $Z_{4}$ due to all Zernike modes present over the metapupils for NGS and LGS respectively. Focus combination on the two DMs is badly seen. In effect the ratio on the LGS footprint between a focus and a tilt introduced by a focus on the metapupil, is constant and does not depend on the layer height. Using $Z_{4}, Z_{5}$ and $Z_{6}$ measurements on a NGS having a different footprint geometry permits independent focus measurements.

So we add a new matrix block in the IM, corresponding to the NGS propagation. We then consider that we have a new WFS pointed on the NGS. Finally the information from NGS is used to measure isoplanatic TT (unseen from LGS's) [3], [7]. Its measurements are added to LGS measurements, in order to be multiplied by the reconstruction matrix. The results give us commands to send to DM's that will correct all Zernike modes starting from $Z_{2}$.

\section{Results for ELT's}

In this section we present results for an MCAO correction starting from $Z_{2}$ with the configuration detailed in Sec.2.

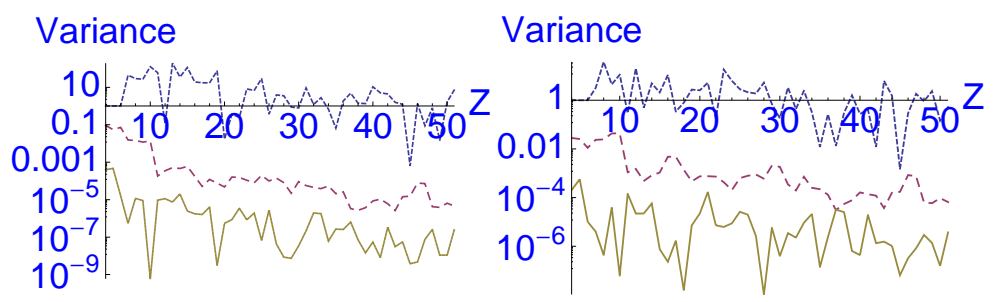

Fig. 4. Residual Error with NGS / 2DM - 2L. The plot shows the residual error variance after correction (using 4 LGS's and 1 NGS for LO modes) in $\mathrm{rad}^{2}$ on a logarithmic scale vs Zernike mode number. The top dashed lines represent Zernike coefficients of the atmospheric turbulence, solid lines the residual error variance after correction. The large dashed line is the residual shown on Fig.1. Left and right plots are for the DM's conjugated respectively at $1 \mathrm{~km}$ and $8 \mathrm{~km}$.

Fig. 4 shows the residual error variance after correction when using our technique. The contribution of the NGS (different footprints dimension) permits to overcome the problem explained in Sec.5. We notice the behavior of the residual error variance is following behavior of input coefficients. For the 
first 50 modes the residual error variance meaned is of $2.5 * 10^{-5} \mathrm{rad}^{2}$. We extrapolate the results to a 2000 modes correction case by summing this average value in quadrature. The final results is a variance of $5.1 * 10^{-2} \mathrm{rad}^{2}$ at the Sodium Laser wavelength. This value corresponds to a variance of $6.8 * 10^{-3} \mathrm{rad}^{2}$ in $\mathrm{H}$ Band $(\mathrm{SR}=0.99)$.

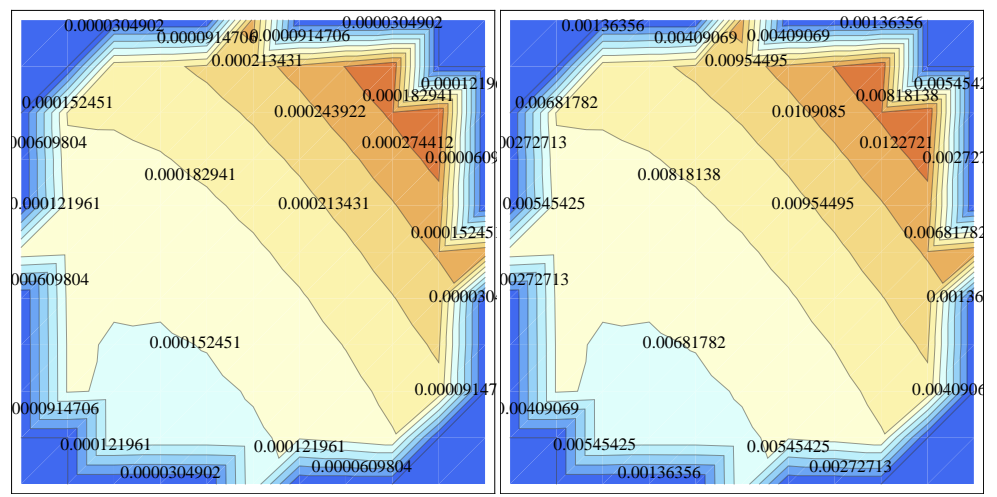

Fig. 5. The left plot represents the residual error variance of MCAO correction over the 2 arcmin FoV, simulated for 50 Zernike modes. Right plot is the extension of the left one, calculated for 2000 Zernike modes at the Sodium Laser wavelength.

On FIg.5, left plot, we see the residual error variance over the FoV for 50 Zernike modes. This corresponds to the residual error between the MCAO estimate wavefront in a given FoV direction, and the wavefront perturbation encounterd from a NGS located in that particular direction. One can notice it is pretty homogeneous. The total mean of the residual error variance over the 2 arcmin FoV is of : $0.2 * 10^{-3} \mathrm{rad}^{2}$.

On the right plot, is shown the residual error variance scaled from 50 to 2000 corrected modes over the FoV. The residual is negligeable with respect to other source of errors in the MCAO system. This is a first check of the validity of the described technique.

\section{Discussion \& Conclusion}

Simulations presented in this paper have been done supposing the LGSS to be launched from inside the telescope pupil. In a more realistic case, the LGS projectors are located behind the secondary mirror. In such a configuration (see Fig.6)the shadow projected by the obstruction prevents to properly sample the upward propagation of the LGS. Indeed for each LGS, on Fig.6, the shadow zone which is not covered by the other 3 LGS's and encountered by the upward beam is relatively small. Future studies will be done to quantify the effect of telescope central obscuration and the best placing of the laser projectors behind the secondary.

Another interesting point we noticed during this work is that it is not possible, using one type of star, to correctly re-distribute a Zernike mode over a number of layers greater than the radial order $n$ of the mode. The demonstration of the concept is the object of a future paper. It is a generalization of the problem described in Sec.5 [3], [7]. Finally the present paper shows that for LGS MCAO system it is possible to use a different form of IM. This form includes the effect of the laser upward propagation in the LGS wavefront sensing process. With this IM the MCAO reconstructor is generating a lower reconstruction error than a classical MCAO reconstructor that discard the LGS Tilt information. This is true in the case of reconstruction limited to modes higher then $Z_{6}$ in the considered case of a two layers and two DM's. Then we described how using the measurements of $Z_{2} ; Z_{3} ; Z_{4} ; Z_{5} ; Z_{6}$ obtained by observing a NGS found in the MCAO FoV, together with the LGS measurements. This improve the MCAO reconstruction of modes from $Z_{2}$ to $Z_{6}$. In the case of $2 \mathrm{DMs}$ and 2 atmospheric layers our 
numerical results showed that the technique allows to retrieve the atmospheric turbulence with total residual error variance of $1.7 * 10^{-4} \mathrm{rad}^{2}$ in $\mathrm{H}$ band for the first 50 modes. This result has been extrapolated to 2000 modes, obtaining a residual variance of $6.7 * 10^{-3} \mathrm{rad}^{2}$ in $\mathrm{H}$ band $(\mathrm{SR}=0.99)$. Again it is to be noted that the applied technique works for an MCAO reconstructor having 2 DMs. Future work will have the aim to: 1) generalize to 3 layers 2) compute performance when measurements noise is considered 3) directly compute the error for a high number of modes.

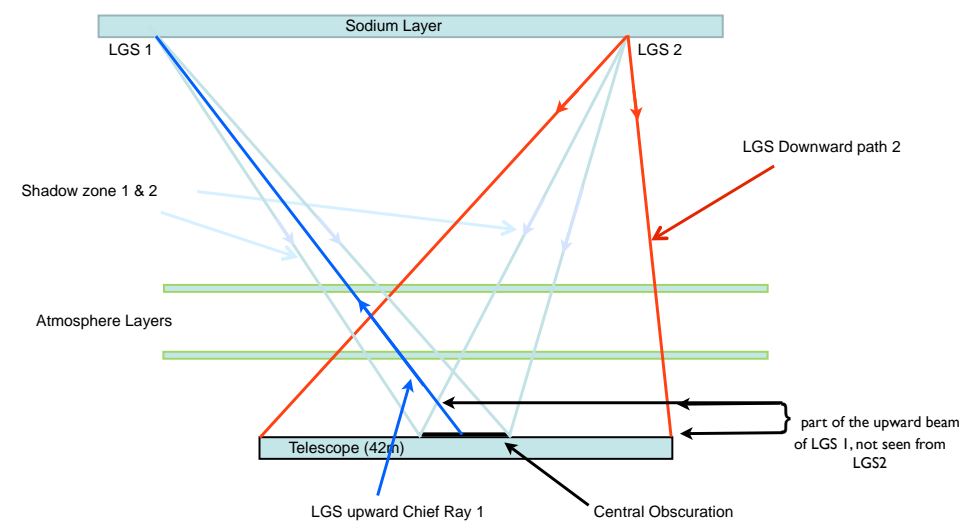

Fig. 6. Central Obscuration. The Chief Ray of LGS 1 is upward-propagating inside the shadow zone, unseen by LGS 1 during its downward propagation. However only a small part of the upward path of LGS 1 is not seen during the downward propagation of LGS 2 .

\section{References}

1. Bahcall, J.N. and Soneira, R.M., The Astrophysical Journal The distribution of stars to $\mathbf{v}=\mathbf{1 6}$ th magnitude near the North Galactic Pole: Normalization, custering properties, and counts in various bands, (1981), vol 246, p. 122-135

2. Beckers, J.M., A\&A Adaptive Optics for astronomy: Principles, Performance, and Applications, (1993), vol.31 p. 13-62

3. Brusa, G. et al., SPIE proceeding A study for a multi-conjugate AO system for 8m class telescope, (2000), vol. 4034, p. 190-200

4. Clare, M.C. and Ellerbroek, B.L. J. Opt. Sky coverage estimates for adaptive optics systems from computations in Zernike space, (2006), vol.23, p. 418-426

5. Conan, J.M. et al., J. Opt. Wave-front temporal spectra in high-resolution imaging through turbulence, (1995), vol. 12, No. 7, p. 1559 - 1570

6. Ellerbroek, B.L. et al., SPIE proceeding MCAO for Gemini-South, (2003), vol. 4839, p. 55-66

7. Femenìa, B., J. Opt. Soc. Am. A Tip-Tilt reconstruction with a single dim natural guide star in mutliconjugate adaptive optics with laser guide stars, (2005), vol. 22, p. 2719-2729

8. Lombini, M. et al., SPIE proceeding Preliminary design of the post focal relay of the MCAO module for the E-ELT, (2008), vol. 7015, p. 1-7

9. Noll, R. J., Optical Society of America Zernike polynomials and atmospheric turbulence,(1976), vol. 66, p. 207-211

10. Ragazzoni, R. A\&A Modal tomography for adaptive optics, (1999), vol. 342, p.53-56

11. Ragazzoni, R. Laser Guide Star Advanced Concepts: tilt Problem, (2000), 2000lgsa.conf..125R

12. Rigaut, F. et al., SPIE procceding Principles, limitations, and performance of multiconjugate adaptive optics, (2000), vol. 4007, p. 1022-1031 